\title{
Sistematización de experiencias como método para la retroalimentación de la práctica educativa
}

\author{
Systematization of experiences as a method for feedback on \\ educational practice
}

'Teresa de Jesús Barreras Villavelázquez, ${ }^{2} \mathrm{Ma}$. Concepción Soto Valenzuela, ${ }^{3}$ Wilberth Velducea Velducea, ${ }^{4}$ Rigoberto Marín Uribe, ${ }^{5}$ Pamela Franco Díaz, ${ }^{6}$ Azaneth Laguna Celia \& ${ }^{7}$ Isabel Guzmán Ibarra

\begin{abstract}
Barreras, T., Soto, M. C., Velducea, W., Marín, R., Franco, P., Laguna, A., \& Guzmán, I. (2021). Sistematización de experiencias como método para la retroalimentación de la práctica educativa. Revista Ciencias de la Actividad Física UCM, 22(2), julio-diciembre, 1-17. https://doi.org/10.29035/rcaf.22.2.6
\end{abstract}

\section{RESUMEN}

El proceso de enseñanza-aprendizaje hace prioritario valorar las experiencias y perspectivas de los actores involucrados, para ello, es necesario promover la dialogicidad entre estudiantes y docentes, como una actividad de retroalimentación que oriente a la reflexión y análisis en busca de la adaptación y mejora del mismo. El objetivo del estudio fue valorar la práctica educativa sustentada en el enfoque de pensamiento complejo, a partir de la perspectiva estudiantil. El presente trabajo muestra los datos obtenidos a partir de sistematización de experiencias académicas desarrollada con 23 estudiantes del segundo semestre de Licenciatura en Educación Física, con respecto, al primer objeto de estudio del proyecto formativo implementado que atiende la asignatura Bases Psicopedagógicas en el semestre enero-junio 2018, para lo cual se atendieron tres fases procedimentales: reconstrucción, interpretación y potenciación de la experiencia. Haciendo uso de la videograbación y observación participante, se analizó como unidad de observación al pensamiento complejo y como unidades de análisis 1. La planeación analítica de clase compuesta por 2. Estrategias docentes innovadoras y 3. La metodología de evaluación. Los resultados muestran que los estudiantes manifiestan interés y motivación por las unidades de análisis 2 y 3, expresando que les favorecen las clases dinámicas y la valoración de su trabajo con un carácter formativo y no memorístico. En cuanto a la unidad de observación 1, manifestaron un dominio limitado acerca de la propuesta presentada por la docente. Se rescata su interés y satisfacción porque las asignaturas teóricas los sitúen en su realidad laboral.

Palabras clave: educación física, sistematización de experiencias, práctica educativa, universitarios, videograbación.

\footnotetext{
'Maestría en Psicomotricidad. Facultad de Ciencias de la Cultura Física, Universidad Autónoma de Chihuahua. Chihuahua, México. tbarreras@uach.mx|https://orcid.org/0000-0002-4241-3810

2 Doctorado en Ciencias de la Cultura Física. Facultad de Ciencias de la Cultura Física, Universidad Autónoma de Chihuahua. Chihuahua, México. masoto@uach.mx | http://orcid.org/0000-0002-1043-7631

${ }^{3}$ Doctorado en Ciencias de la Cultura Física. Facultad de Ciencias de la Cultura Física, Universidad Autónoma de Chihuahua. Chihuahua, México. wvelducea@uach.mx | https://orcid.org/0000-0001-8059-0706

${ }^{4}$ Doctorado en Ciencias de la Educación. Facultad de Ciencias de la Cultura Física, Universidad Autónoma de Chihuahua. Chihuahua, México. rmarin@uach.mx | https://orcid.org/0000-0002-8672-4047

5 Maestría Profesional en Psicomotricidad. Facultad de Ciencias de la Cultura Física, Universidad Autónoma de Chihuahua. Chihuahua, México. pfranco@uach.mx | https://orcid.org/0000-0002-7148-8715

${ }^{6}$ Maestría Profesional en Psicomotricidad. Facultad de Ciencias de la Cultura Física, Universidad Autónoma de Chihuahua. Chihuahua, México. alaguna@uach.mx | https://orcid.org/0000-0001-8017-4104

${ }^{7}$ Doctorado en Ciencias de la Educación. Facultad de Filosofía y Letras, Universidad Autónoma de Chihuahua. Chihuahua, México. iguzman@uach.mx | https://orcid.org/0000-0001-6075-5024
} 
Barreras, T., Soto, M. C., Velducea, W., Marín, R., Franco, P., Laguna, A., \& Guzmán, I. (2021). Sistematización de experiencias como método para la retroalimentación de la práctica educativa. Revista Ciencias de la Actividad Física UCM, 22(2), julio-diciembre, 1-11. http://doi.org/10.29035/rcaf.22.2.9

\section{ABSTRACT}

In the teaching-learning process it becomes a priority to assess the experiences and perspectives of the actors involved, for this it is necessary to promote dialogue between students and teachers, as a feedback activity that guides reflection and analysis in search of adaptation and of the improvement of it. The objective was to assess the educational practice based on the complex thinking approach from the student perspective. The present work shows the data obtained from Systematization of Academic Experiences developed with 23 students of the second semester of the Degree in Physical Education, with respect to the first object of study of the formative project implemented that attends the subject Psychopedagogical Bases in the semester January -June 2018, for which three procedural phases were attended: reconstruction, interpretation and enhancement of the experience. Using video recording and participant observation, it was analyzed as a unit of observation to complex thinking and as units of analysis 1. The analytical class planning, 2. The teaching strategies implemented and 3. The evaluation methodology. The results show that the students express interest and motivation for the analysis units 2 and 3, expressing that they favor dynamic classes and the valuation of their work with a formative and nonmemorial character. Regarding the observation unit 1, they expressed limited mastery of the proposal presented by the teacher. Their interest and satisfaction are rescued because the theoretical subjects place them in their work reality.

Key words: Physical education, systematization of experiences, educational practice, university students, videotape.

\section{INTRODUCCIÓN}

La labor docente implica diversos desafíos entre los cuales se encuentran atender las necesidades de los estudiantes y sus contextos, es decir, situar el proceso formativo en los escenarios reales. Balladares et al. (2016), cuestionan los retos contemporáneos del educador en materia de innovación, así como en el uso de herramientas digitales; consideran que entre las problemáticas de la educación, la de mayor notoriedad se refiere al énfasis que existe sobre la enseñanza cognitiva por encima de la interiorización de aspectos procedimentales y actitudinales, generando con ello consecuencias evidentes tanto en los procesos formativos como en el desarrollo de pensamiento crítico y reflexivo en los estudiantes (Velducea et al., 2019).

Lo anterior se ve reflejado en la continuidad y aceptación de prácticas lineales en las que el docente asume una función directiva y el estudiante se limita a la recepción de conocimientos, enmarcados en un paradigma tradicional, estableciendo con ello que la participación y autorregulación del estudiante sobre su proceso de aprendizaje no es necesaria, mucho menos enriquecedora. Pero ¿qué pasaría si la práctica pedagógica atendiera a la construcción docente como ser humano y como parte de una comunidad en la que más que transferir conocimientos puros, contribuya al desarrollo de la cultura en el contexto educativo?

Rey \& Marmolejo (2019) consideran que, en la actualidad, el trabajo académico puede y debe desenmarcar el rol absolutista y tradicional del docente a través del desarrollo e intervención de diversas estrategias didácticas, adaptadas a las necesidades de los estudiantes y las características del medio en el que se desenvuelven. La contextualización de la práctica es la que otorga al estudiante elementos de análisis y síntesis para la adquisición de aprendizajes significativos, por lo que el proceso debe ser acompañado y orientado desde la creatividad.

Los estudiantes del área de cultura física, durante todo su proceso formativo, interactúan con una diversidad de contextos sociales y de problemáticas propias de los mismos. Por lo que el acompañamiento docente desde una mirada innovadora, parte de la actualización curricular para 
Barreras, T., Soto, M. C., Velducea, W., Marín, R., Franco, P., Laguna, A., \& Guzmán, I. (2021). Sistematización de experiencias como método para la retroalimentación de la práctica educativa. Revista Ciencias de la Actividad Física UCM, 22(2), julio-diciembre, 1-11. http://doi.org/10.29035/rcaf.22.2.9

el desarrollo de la autonomía y responsabilidad en los estudiantes universitarios. Es esencial considerar como temática de dicho proceso, el uso de estrategias para la educación en valores que permitan la participación del grupo en el proceso formativo. La intervención a partir de técnicas participativas y métodos de enseñanza en los que se plantean actividades científicas dan valor a la responsabilidad y autonomía del estudiante. Lo anterior con base en la construcción socio cultural de la figura docente (López, 2020).

El docente debe reconocer a sus estudiantes y empatizar con ellos, comprendiendo que son personas interesadas en formarse y que, por tanto, complementan sus prácticas académicas en la institución escolar a través de diversos medios. Es común que los estudiantes universitarios se apoyen en el uso de las tecnologías de la información para responder a problemáticas o necesidades entre las que se encuentran sus procesos pedagógicos. El uso de diversos medios en imagen, texto, videos, etc., gestiona la asimilación de la información planteada desde un proceso investigativo del que parte la innovación, en este caso, generalmente asociada a la actualización de los docentes en su proceso de enseñanza, sin embargo, no implica obligatoriedad en el uso de las tecnologías. La innovación, parte de la búsqueda de experiencias reveladoras que puedan ser replicadas en escenarios reales (Escudero et al., 2019).

Existen tres áreas en las que se suscriben las prácticas de docencia universitaria: el currículo, la acción educativa y procesos sociales involucrados. El currículo, establece las adquisiciones epistemológicas del área académica, así como las experiencias prácticas necesarias para su dominio, guiándose por concepciones, retos y referentes institucionales; mientras que la práctica o acción educativa y los aspectos sociales; hacen alusión a la articulación de todos los elementos del proceso educativo como: estrategias de enseñanza, estrategias de aprendizaje, contrato didáctico, dinámica o clima de aula con los vínculos sociales que se generan entre los autores involucrados, reconociendo que el quehacer docente implica un diálogo crítico y reflexivo que dé significado a su labor, que mientras más íntimo sea el enlace y más consiente la reflexión, más genuina será la aportación cultural y atención al ser humano en el proceso educativo (Domingo, 2019).

Todos estos factores involucrados en la enseñanza-aprendizaje, dirigen a la necesidad de valorar las experiencias y perspectivas de estudiantes y docentes, con la intención de promover la dialogicidad como una actividad de retroalimentación que oriente a la reflexión y análisis en busca de la adaptación y de la mejora del proceso educativo, mientras que su calidad se encuentra vinculada con la contribución que otorga la exploración de experiencias, maximizando su utilidad y potencial, si se desarrolla una sistematización y, más aún, si ésta forma parte de un proyecto de investigación.

La Sistematización de Experiencias, es un método que pretende comprender, tanto la intervención como el aprendizaje, en beneficio de las prácticas educativas. Messina \& Osorio (2016) plantean que sistematizar implica procesos inclusivos y participantes en la investigación, comprendiéndola como una investigación narrativa, en la que los relatos se convierten en esencia y medio para el conocimiento. A través de ella es posible recuperar, construir e interiorizar el proceso formativo desde un enfoque colaborativo en el que se vinculan los referentes históricos de cada uno de los participantes; asume una postura crítico-reflexiva que se adapta a las características de los participantes y de la experiencia con respecto a la temporalidad y contexto (Barbosa \& Barbosa, 2017). Por lo anterior, el objetivo fue valorar la práctica educativa sustentada en el enfoque de pensamiento complejo a partir de la perspectiva estudiantil.

\section{METODOLOGÍA}

El presente trabajo muestra los datos obtenidos a partir de una Sistematización de Experiencias académicas desarrolladas con 23 estudiantes del 
Barreras, T., Soto, M. C., Velducea, W., Marín, R., Franco, P., Laguna, A., \& Guzmán, I. (2021). Sistematización de experiencias como método para la retroalimentación de la práctica educativa. Revista Ciencias de la Actividad Física UCM, 22(2), julio-diciembre, 1-11. http://doi.org/10.29035/rcaf.22.2.9

segundo semestre de Licenciatura en Educación Física, con respecto al primer objeto de estudio del proyecto formativo implementado que atiende la materia Bases Psicopedagógicas en el semestre enero-junio de 2018.

El proceso metodológico se enmarca en una investigación del enfoque cualitativo, con el diseño de Investigación Acción Participativa y con el método de estudio de caso narrativo y la Sistematización de Experiencias (Jara, 2018, Pastor \& Espeso, 2015, Guevara et al., 2020). Se atendieron las tres fases procedimentales planteadas por Sánchez (2010), para la recreación de la experiencia: reconstrucción, interpretación y potenciación de la experiencia.

Como unidad de análisis se estableció al pensamiento complejo descrito por Escalona \& Torres (2015), como la capacidad de asociar los diversos referentes de la realidad con su trabajo profesional, para abordar contextos y necesidades que la sociedad demande por medio de la reflexión y adaptación. Y como unidades de observación: unidad 1, la planeación analítica de clase, conceptualizada como la descripción específica y organizada de estrategias de EnseñanzaAprendizaje (E-A), con el objetivo de movilizar saberes; unidad 2, las estrategias docentes innovadoras comprendidas como los procedimientos o recursos utilizados por los docentes para lograr aprendizajes significativos en los estudiantes; y, finalmente, unidad 3, la metodología de evaluación como un proceso secuencial y estructurado de valoración de aprendizajes (Rodríguez \& Toro, 2018).

Se utilizó la observación participante y videograbación para la recolección de datos como técnicas e instrumentos de acuerdo con Barbosa et al. (2017) y el Software Atlas.ti versión 7.5 para el análisis de los mismos. El procedimiento de la investigación consistió en tres etapas: la primera, hace alusión al consentimiento informado de los participantes, en este caso los estudiantes, atendiendo las consideraciones éticas de investigación; la segunda, corresponde al diseño e implementación del proyecto formativo; y la tercera etapa, que consistió en la sistematización de la práctica educativa.

\section{RESULTADOS}

Los resultados obtenidos, muestran que los estudiantes manifiestan mayor interés y agrado por la unidad de observación 2. Estrategias docentes innovadoras, expresando que les agradan más las clases dinámicas, resaltando la importancia del dinamismo en clase a partir de la implementación de actividades lúdicas, pues les permite atender a su proceso de aprendizaje de manera positiva, debido a que se encuentran motivados en las sesiones y son capaces de visualizar las estrategias didácticas en su práctica real como futuros profesionales de educación física. Consideran que la retroalimentación que se llevó a cabo como un diálogo reflexivo en triadas, es una estrategia que los invita a participar, conocerse mejor y trabajar de forma colaborativa, tal como se refleja en los siguientes extractos narrativos y en el Esquema 1:

...Hace las clases dinámicas y eso está padre $[S 7] \ldots$

...Porque en realidad esta clase es teórica $y$ eso es aburrido, y con las diversas dinámicas hemos aprendido más [S7]...

...Ponemos más atención, que si solo estuviéramos sentados [S2]...

...Sí, yo creo que la dinámica es algo más importante, porque estamos estudiando para educación física, para enseñar eso, lo que más nos llama la atención es tener una clase didáctica [S8]...

...Es que es bastante dinámico, han sido juegos, también la parte de la retroalimentación al final de la clase sirve de mucho, este el mapa conceptual, el glosario, todo eso es retroalimentar, retroalimentar y de diferentes maneras, ahi es cuando se hace más dinámica la clase y está bastante bien [S7]... 
Barreras, T., Soto, M. C., Velducea, W., Marín, R., Franco, P., Laguna, A., \& Guzmán, I. (2021). Sistematización de experiencias como método para la retroalimentación de la práctica educativa. Revista Ciencias de la Actividad Física UCM, 22(2), julio-diciembre, 1-11. http://doi.org/10.29035/rcaf.22.2.9

...Nos hace que participemos más, el que al final de la clase nos pregunten qué aprendimos, hace que participemos, de alguna manera, o igual, el otro día que estábamos jugando con los conceptos todos participábamos [S9]...

...También nos enseña mucho a trabajar en equipo, al menos eso me ha servido mucho a mi a trabajar [STO]...

...Incluso innovar, por ejemplo, yo creo que, a mí, me parece, se me hace más interesante la forma dinámica que estar escribiendo, se me hace que siempre hay maneras diferentes de cómo enseñar y todo [S77]...
..."De cierto modo lo que está haciendo la maestra es lo que generalmente se le pide a un maestro, que su clase sea atractiva, que su forma de dar la clase sea algo que atraiga a los estudiantes y los meta en la clase, porque es muy sencillo o se podría decir que es muy sencillo para un maestro llegar y dar instrucciones, hagan un resumen, una síntesis, un ensayo, $x$ cosa de manera individual y me lo entregan y el maestro se sienta se pone a hacer $x$ cosa, y la maestra lo que hace es que siempre hacemos algo, con las dinámicas que ella nos pone sabe cómo involucrarnos a todos" [S72]...

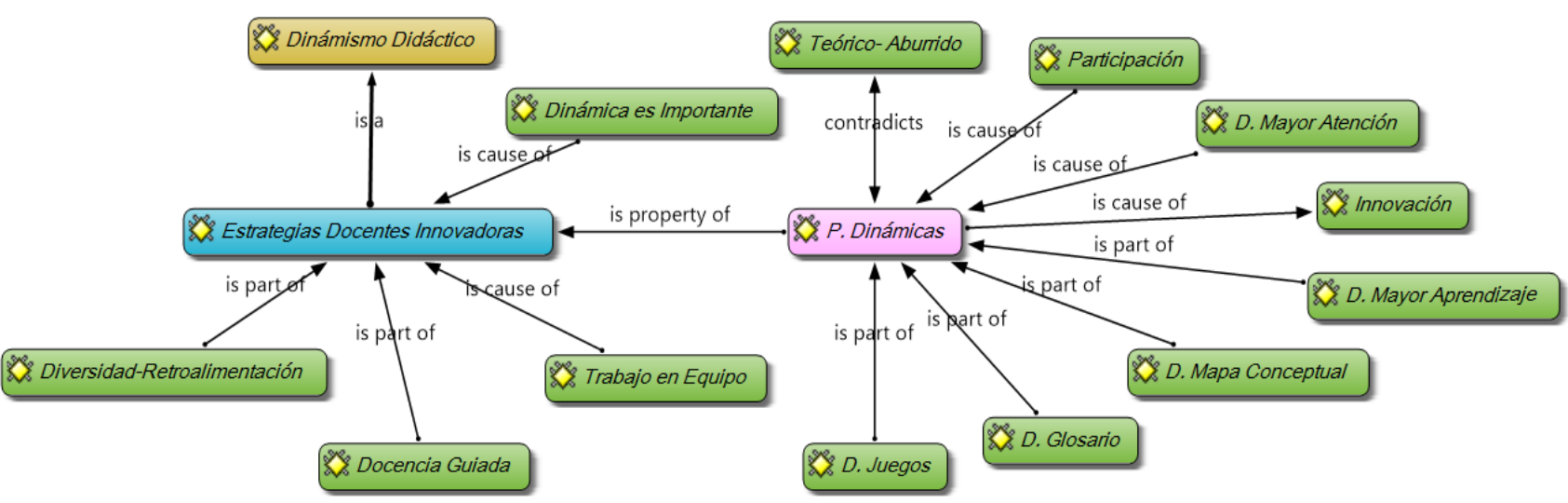

Esquema 7. Estrategias docentes innovadoras.

Así mismo, se presentan comentarios favorables para la unidad 3. Metodología de evaluación estableciendo que la rúbrica como instrumento de valoración de aprendizajes. Es evidente cómo para este grupo el autoevaluarse y co-evaluar a sus compañeros implica responsabilizarse de su proceso de aprendizaje, interiorizar los saberes con una intensión real de aplicación y ejecución en su realidad como estudiante de licenciatura y a futuro, comprometiéndose tanto con su proceso como con el de los integrantes del grupo; dándole, además, un valor a su trabajo con un carácter formativo y no memorístico, como se puede reflejar en la narrativa siguiente y en el Esquema 2:

...A mi me gustó la manera de evaluación pues porque creo que es más abierto y como reflexivo para y entre nosotros, es más reflexivo que simplemente venir hacer un examen y exactamente el concepto que se tiene en un libro, que exactamente el concepto que tiene en internet, en cambio ya es más propio tuyo y lo puedes utilizar mejor a futuro, es lo que me gusta de la evaluación, a lo mejor y si es bueno de vez en cuando 
Barreras, T., Soto, M. C., Velducea, W., Marín, R., Franco, P., Laguna, A., \& Guzmán, I. (2021). Sistematización de experiencias como método para la retroalimentación de la práctica educativa. Revista Ciencias de la Actividad Física UCM, 22(2), julio-diciembre, 1-11. http://doi.org/10.29035/rcaf.22.2.9

meter una evaluación escrita para a lo mejor saber exactamente el nivel de aprendizaje de cada uno, sin embargo, si me gusta más así como lo hicimos [S7]...

...Además, la maestra nos hace ver que no necesitamos de un examen para aprender [S13]...

...Es que el examen no garantiza que hayas aprendido es saber que tienes más que nada en algunos, buena memoria, porque si se fijan es mucho estar repasando, repasando y repasando, pasa el examen y ya se les olvido todo, si es más dinámico hacerlo así, al momento de ver algún concepto te acuerdas ah, yo hice esta actividad y fue este concepto, es más práctico [S74]...

...Te obliga aprender, porque un examen, sabes que tendrás el examen y es memorizarlo y hacerlo y acá sabes que no vas a tener examen, es realmente de saberlo [S75]..

...Y luego también que tuvimos que decir lo que creíamos habíamos aprendido o habíamos cumplido con los aprendizajes de la materia, y valorar lo de nuestros compañeros con los mismos criterios, eso te obliga, jajaja, bueno tienes que ser real $[S 4] \ldots$

...Pues es la clásica diferencia entre una materia teórica y una materia más práctica, que en la teórica puedes estar viendo o haciendo muchos trabajos durante la unidad y al final ya que termines el examen vas a hacer lo mismo siempre, porque eso no es bueno solo leer, leer, leer, para aprenderse lo que va a venir en el examen y luego en la segunda unidad cuando vas en teoría avanzada pues eso ya no lo vas a volver a ver, en cambio si es dinámica la clase, te acuerdas de un dibujo o de un compañero, lo que dialogaron y así cosas que a ti te llamaron la atención [S76]...

...En la actividad de las imágenes estuvo padre porque era identificar el concepto a como tú te lo sabias y ya cuando lo aterrizamos a un crucigrama era como la retroalimentación para saber cuál era el verdadero concepto, si lo aprendiste o no $[S 7] \ldots$

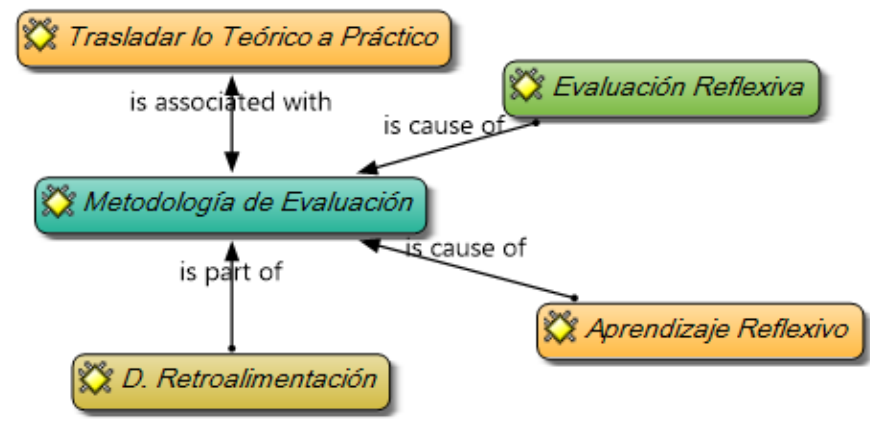

Esquema 2. Metodología de evaluación.

En cuanto a la unidad de análisis 1. Planeación de clase, los estudiantes mostraron menor interés, desconocimiento o falta de dominio con respecto a los elementos que se establecieron en la propuesta de planeación, la cual incluía: objeto de estudio, presentación, situación problema, actividades de aprendizaje, evidencia de desempeño, recursos de apoyo, dispositivo de evaluación y referencias 
Barreras, T., Soto, M. C., Velducea, W., Marín, R., Franco, P., Laguna, A., \& Guzmán, I. (2021). Sistematización de experiencias como método para la retroalimentación de la práctica educativa. Revista Ciencias de la Actividad Física UCM, 22(2), julio-diciembre, 1-11. http://doi.org/10.29035/rcaf.22.2.9

bibliográficas, de acuerdo al dispositivo de formación planteado por Guzmán et al. (2014). Por ello, las aportaciones fueron menores expresando un dominio limitado acerca de la propuesta presentada por la docente, hubo escasos comentarios con respecto al contenido, de los cuales, es posible rescatar su interés y satisfacción porque las asignaturas teóricas los sitúen en su realidad laboral, tal como se muestra en los extractos narrativos siguientes y en el Esquema 3:

...Yo creo que es posible que podamos replicar el tipo de planeación a nuestra práctica, utilizando además otros métodos de enseñanza que hemos visto creo que si nos podrían servir [S7]...

...Se tienen que unir tanto los objetivos del maestro como del alumno en un plan de estudio o perfil de egreso, en el que se supone que, con este nuevo plan de trabajo de las universidades, en el que ya vieron cuales son las competencias que debe tener un educador físico, con cuáles competencias debe de salir, entonces cada objetivo de cada maestro debe ser para que también el alumno pueda tener esas competencias determinadas para impartir clases en un futuro, yo creo eso [S2O]...

...Es bueno que se incluya la retroalimentación pues además te motivas y te permite que se dé 10 dinámico [S76]...

... Una clase teórica hacerla practica está súper bien, la verdad yo ya había preguntado de esta clase y me habian dicho: no esa materia está muy aburrida te vas a dormir y la verdad es que, a mí, esas clases me desesperan o el estar mucho tiempo sentado pues si desespera, y ya de que la maestra algunas veces nos para a hacer una actividad hasta de despierta de cierta forma [S7]...

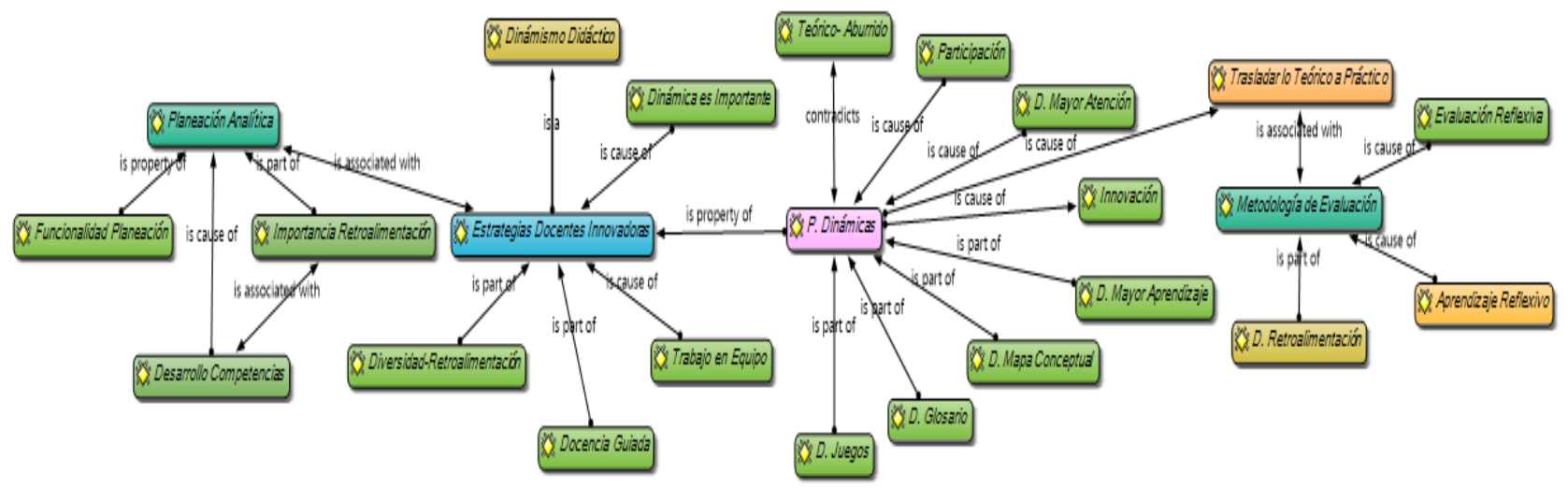

Esquema 3. Planeación de clase.

En el Esquema 4 se presenta la vinculación entre la unidad de análisis y la unidad de observación. Se encontró una asociación entre la planeación de clase y las estrategias docentes innovadoras, así como una perspectiva positiva de las dinámicas aplicadas por la docente para la transición de lo teórico a lo práctico. Se muestra agrado por parte de los estudiantes en los procesos de comunicación, colaboración, aprendizaje y evaluación que se llevaron a cabo, así como análisis de los objetivos de las actividades o estrategias didácticas aplicadas. Finalmente, uno de los estudiantes comentó como propuesta o aportación para mejorar el vínculo con la docente y los procesos de aprendizaje lo siguiente: 
Barreras, T., Soto, M. C., Velducea, W., Marín, R., Franco, P., Laguna, A., \& Guzmán, I. (2021). Sistematización de experiencias como método para la retroalimentación de la práctica educativa. Revista Ciencias de la Actividad Física UCM, 22(2), julio-diciembre, 1-11. http://doi.org/10.29035/rcaf.22.2.9

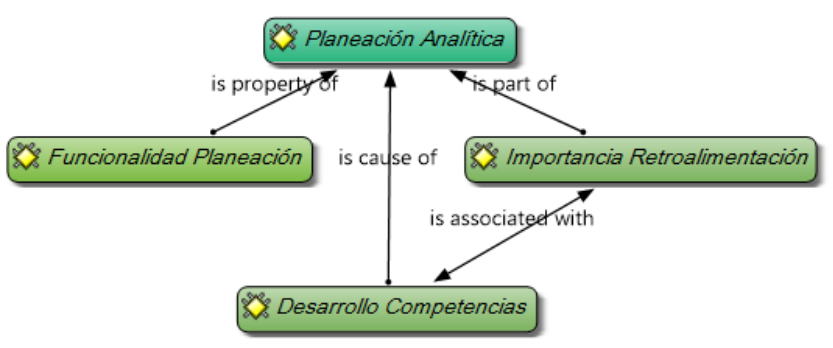

Esquema 4. Implementación del enfoque de pensamiento complejo.

...A lo mejor también, al momento de que hacemos los juegos que Ud. también jugara o que por ejemplo a la hora de los roles que Ud. también de repente se juntara con un equipo y Ud. jugara un rol o un turno para que nos quedara más

\section{DISCUSIÓN}

En la actualidad el enfoque de pensamiento complejo establece la necesidad de un acompañamiento que respete los recursos personales de quienes se encuentran inmersos en un proceso formativo, de tal manera que se comprenda a la práctica docente y a la intervención educativa como un sistema holístico, en el que se vuelve primordial atender necesidades, intereses educativos, contextos y experiencias de vida. Desde la concepción de Morín (1999), somos responsables de comprender que todos las interacciones y eventos del individuo constituyen un fenómeno social, pero que, a su vez, cada uno de dichos elementos es también un fenómeno, siendo importante el estudio de la particularidad y totalidad de los fenómenos. Lo anterior permite una práctica docente contextualizada.

La docencia en el nivel universitario es comúnmente una formación en servicio producto de la experiencia o de procesos de capacitación y/o actualización, por lo que es necesario ubicar al docente en un paradigma en el que se convierta en un practicante reflexivo para atender las demandas sociales y contextuales de los estudiantes (Guzmán claro, porque por ejemplo yo mire que, al momento de realizar roles, si me quedaban dudas de cómo es mi rol en el diálogo reflexivo, si se integra $\mathrm{Ud}$. a 10 mejor nos queda más claro el rol [S7]...

et al., 2012). Existe una relación intrínseca entre la planeación analítica, las estrategias docentes y la metodología de evaluación con el desarrollo del proceso enseñanza-aprendizaje.

La experiencia videograbada en los estudiantes refleja uno de los mayores problemas que se viven en la práctica educativa: la falta de interés que le dieron a la planeación de la clase; esto podría explicar que, si desde que el docente en formación no le otorga el valor que corresponde a planear su práctica educativa, posiblemente no lo hará cuando se encuentre en su ejercicio profesional. Para ello, la Organización de las Naciones Unidas para la Educación, la Ciencia y la Cultura (UNESCO, 2016), menciona que, para participar en una experiencia significativa, ésta debe de ser concreta con el fin de desarrollar aprendizajes significativos, situada en un espacio y tiempo determinados, con acciones y actividades identificables; por lo que la planeación es inherente a ello. Debe estar contextualizada porque planea sus acciones en estrecha relación con el medio cultural, social y político y las necesidades de desarrollo de los estudiantes a quienes atiende. 
Barreras, T., Soto, M. C., Velducea, W., Marín, R., Franco, P., Laguna, A., \& Guzmán, I. (2021). Sistematización de experiencias como método para la retroalimentación de la práctica educativa. Revista Ciencias de la Actividad Física UCM, 22(2), julio-diciembre, 1-11. http://doi.org/10.29035/rcaf.22.2.9

En cuanto a las estrategias docentes, Méndez \& Conde (2018) señalan que los estudiantes universitarios exigen que la metodología de enseñanza sea dinámica, participativa, amena y flexible. Con lo que sus aportaciones destacan la necesidad de tareas propiamente prácticas que propicien su participación y protagonismo en el trabajo áulico.

Por su parte, Montanares \& Junod (2018), indican que los docentes enfatizan en la administración de contenidos para que los estudiantes logren un aprendizaje independientemente de las estrategias seleccionadas. Aun cuando concuerdan con que la participación de los estudiantes es de gran valor, dan prioridad a las sesiones expositivas.

\section{CONCLUSIONES}

La Sistematización de Experiencias como práctica educativa e investigativa, permite un acercamiento docente-estudiante que moviliza adaptaciones sociales en el proceso educativo, ya que facilita reconstruir experiencias, la adaptación y toma de decisiones para que la puesta en práctica del proceso formativo se encause en la autogestión y autorregulación del estudiante. Reconocer la perspectiva de los actores del proceso educativo propicia involucrar los contextos individuales y grupales que se encuentran inmersos directa e indirectamente.

Desde una perspectiva docente, la planeación analítica de la clase es un elemento importante para el desarrollo del proceso de E-A, como un medio de seguimiento en las metas curriculares, para proponer estrategias de movilización de saberes a los estudiantes, compartir recursos de apoyo y procesos de evaluación, entre otros. En el caso de los estudiantes, la planeación en su totalidad o segmentos particulares parece ser relevante cuando encuentran en ella una utilidad en el quehacer de su práctica profesional. En relación a las estrategias docentes, las propuestas dinámicas acompañadas de retroalimentación y practica reflexiva propician una transposición didáctica. Ahora bien, si las estrategias de E-A son innovadoras propician un ambiente de aprendizaje satisfactorio en el que prestan atención a su proceso personal de aprendizaje y al trabajo colaborativo, lo que se evidencia con procesos de escucha activa y participación en las actividades e interiorización de saberes.

Los estudiantes son capaces de romper el paradigma de la evaluación tradicional y el aprendizaje orientado al cumplimiento de una prueba, cuando el docente acompaña y orienta poniendo en el centro del proceso intereses, necesidades, características, dinámicas grupales y sociales. Contextualizar la práctica pedagógica, construir y adaptar el proyecto formativo de manera colaborativa docente-docente, docenteestudiante y estudiante-estudiante, a partir de los referentes, perspectivas y experiencias de cada uno genera el aprendizaje significativo.

Los procesos de comunicación en tríadas y el análisis de los contenidos académicos, favorecen la interacción y colaboración grupal, promoviendo valores sociales como el respeto y la autonomía, así como habilidades de empatía, escucha activa, asertividad, construcción y estructuración de ideas. Es necesario que los docentes universitarios realicemos investigaciones acerca de los modelos pedagógicos y estrategias didácticas que implementamos, como un medio de colaboración y actualización de nuestras prácticas formativas a través de la experiencia reflexiva. 
Barreras, T., Soto, M. C., Velducea, W., Marín, R., Franco, P., Laguna, A., \& Guzmán, I. (2021). Sistematización de experiencias como método para la retroalimentación de la práctica educativa. Revista Ciencias de la Actividad Física UCM, 22(2), julio-diciembre, 1-11. http://doi.org/10.29035/rcaf.22.2.9

\section{REFERENCIAS BIBLIOGRÁFICAS}

Balladares, J., Avilés, M., \& Pérez, H. (2016). Del pensamiento complejo al pensamiento computacional: retos para la educación contemporánea. Sophia, colección de Filosofía de la Educación, 27(1), 143-159. https://doi.org/10.17163/soph.n21.2016.06

Barbosa-Chacón, J., \& Barbosa, J. (2017). La evaluación desde la sistematización de guiones de aprendizaje: Una apuesta en educación virtual. Revista Espacios, 38(60), 20-30.

https://www.revistaespacios.com/a17v38n6 o/17386020.html

Barbosa-Chacón, J., Barbosa, J., \& Villamizar, J. (2017). Aspectos Metodológicos de la Sistematización de Experiencias Educativas (SE): Aportes desde la formación universitaria. Revista Espacios, 38(35),

17-36. https://www.revistaespacios.com/a17v38n3 5/17383517.html

Domingo, A. (2019). La profesión docente desde una mirada sistémica. Revista Panamericana de Pedagogía, (28), 15-35. https://doi.org/10.21555/rpp.v0i28.1618

Escalona, L., \& Torres, E. (2015). Mejoramiento de la calidad de la formación en ingeniería con pensamiento complejo y libros electrónicos didácticos. Revista digital de investigación y postgrado, 5(1), 670-689. https://dialnet.unirioja.es/servlet/articulo?c odigo $=5161343$

Escudero, J., Campillo, M., \& Sáez, J. (2019). El máster de formación inicial del profesorado de educación secundaria revisión, balances y propuestas de mejora. Profesorado Revista de currículum y formación del profesorado, 23(3), 165-188. https://dialnet.unirioja.es/servlet/articulo?c odigo=7185949
Guevara, G.P., Verdesoto, A.E., \& Castro, N.E. (2020). Metodologías de investigación educativa (descriptivas, experimentales, participativas, y de investigación-acción). Recimundo, 4(3), 163-173. https://recimundo.com/index.php/es/articl e/view/860

Guzmán, I., Marín, R., \& Inciarte, A. (2014). Innovar para transformar la docencia universitaria. Universidad del Zulia.

Guzmán, I., Marín, R., Zesati, G. I., \& Breach, R. M. (2012). Desarrollar y evaluar competencias docentes: estrategias para una práctica reflexiva. Voces y silencios. Revista Latinoamericana de Educación, 3(1), 2240 .

https://revistas.uniandes.edu.co/doi/abs/10.1 8175/vys3.1.2012.02

Jara, O. (2018). La sistematización de experiencias: práctica y teoría para otros mundos posibles. CINDE.

López, I. A. (2020). Construcción socio-política de una vocación docente dirigida a la transformación social: el caso de la pedagogía en el campo de la educación física. Revista Ciencias de la Actividad Física UCM, 27(1), 1-74. https://doi.org/10.29035/rcaf.21.1.4

Méndez, J. M., \& Conde, S. (2018). La autorreflexión inicial: una estrategia para la mejora de la práctica docente. Revista Electrónica Interuniversitaria de Formación del Profesorado, 27(1), 17-31. http://dx.doi.org/10.6018/reifop.21.1.270591

Messina, G., \& Osorio, J. (2016). Sistematizar como ejercicio eco-reflexivo: la fuerza del relato en los procesos de sistematización de experiencias educativas. Revista eCurriculum, $\quad 74(2), \quad 602-624$. https://www.redalyc.org/articulo.oa?id=766 46850011 
Barreras, T., Soto, M. C., Velducea, W., Marín, R., Franco, P., Laguna, A., \& Guzmán, I. (2021). Sistematización de experiencias como método para la retroalimentación de la práctica educativa. Revista Ciencias de la Actividad Física UCM, 22(2), julio-diciembre, 1-11. http://doi.org/10.29035/rcaf.22.2.9

Montanares, E., \& Junod, P. (2018). Creencias y prácticas de enseñanza de profesores universitarios en Chile. Revista Electrónica de Investigación Educativa, 20(1), 93-103. https://doi.org/10.24320/redie.2018.20.1.1383

Morín, E. (1999). Introducción al pensamiento complejo. Gedisa.

Organización de las Naciones Unidas para la Educación, la Ciencia y la Cultura. (2016). Texto 3: Metodología de Sistematización de Experiencias Educativas Innovadoras. Serie "Herramientas de apoyo para el trabajo docente". UNESCO. http://docentesinnovadores.perueduca.pe/ wp-content/uploads/2017/06/Texto3sistematizacion.pdf

Pastor-Alfonso, M., \& Espeso-Molinero, P. (2015). Capacitación turística en comunidades indígenas. Un caso de Investigación Acción Participativa (IAP). El Periplo Sustentable, (29),

171-208.

https://www.redalyc.org/articulo.oa?id=193 440418012

Rodríguez, A., \& Toro, V. (2018). Etnografía educativa: estrategias de enseñanza-aprendizaje en el curso de los fundamentos históricos y epistemológicos I del programa de psicología de la Universidad Católica de Pereira. http://hdl.handle.net/10785/5067

Rodríguez Jurado, A. M., \& Toro Acevedo, V. (2018). Etnografía educativa: estrategias de enseñanza-aprendizaje en el curso de los fundamentos históricos y epistemológicos I del programa de psicología de la UCP. Universidad Católica de Pereira. https://repositorioslatinoamericanos.uchile. cl/handle/2250/2664609
Sánchez, A. A. (2010). El artículo sistematización de experiencias: construcción de sentido desde una perspectiva crítica. Revista Virtual Universidad Católica del Norte, (29),

https://www.redalyc.org/articulo.oa?id=194 214466001

Velducea, W., Marín, R., \& Soto, M.C. (2019). Estrategias de intervención y pensamiento complejo en la formación universitaria: revisión sistemática. Revista Publicando, 6(20),

5-11. https://revistapublicando.org/revista/index. php/crv/article/view/1308/pdf_1427

Dirección para correspondencia

Teresa de Jesús Villavelázquez

Maestría en Psicomotricidad.

Facultad de Ciencias de la Cultura Física,

Facultad de Ciencias de la Cultura Física, Universidad Autónoma de Chihuahua.

Chihuahua, México.

Dirección postal: Perif. De la Juventud y Circuito Universitario S/N. Universidad Autónoma de Chihuahua, Facultad de Ciencias de la Cultura Física. Chihuahua, México.

ORCID ID: https://orcid.org/0000-00024241-3810

Contacto:

tbarreras@uach.mx

Recibido: 30-04-2021

Aceptado: 14-07-2021 